\title{
When and How to Perform Genetic Testing for Inherited Colorectal Cancer Syndromes
}

\author{
Patrick M. Lynch, JD, MD
}

\begin{abstract}
The 2013 NCCN Clinical Practice Guidelines in Oncology for Colorectal Cancer Screening address the evaluation and management of patients at risk of inherited susceptibility to colorectal cancer. These patients include the 2 broad categories of patients with familial adenomatous polyposis and its variants, and those with hereditary nonpolyposis colorectal cancer, or Lynch syndrome. This article provides a somewhat personal, yet hopefully evidence-based, approach to the questions of when to test individuals for inherited susceptibility, and how to do so. "When" can be taken to mean "under what circumstance" and "at what age"; this article attempts to speak to each sense of the term. (JNCCN 2013;11:1577-1583)
\end{abstract}

\section{NCCN: Continuing Education}

\section{Accreditation Statement}

This activity has been designated to meet the educational needs of physicians and nurses involved in the management of patients with cancer. There is no fee for this article. No commercial support was received for this article. The National Comprehensive Cancer Network (NCCN) is accredited by the ACCME to provide continuing medical education for physicians.
NCCN designates this journal-based CME activity for a maximum of 1.0 AMA PRA Category 1 Credit(s) TM. Physicians should claim only the credit commensurate with the extent of their participation in the activity.

NCCN is accredited as a provider of continuing nursing education by the American Nurses Credentialing Center's Commission on Accreditation.

This activity is accredited for 1.0 contact hour. Accreditation as a provider refers to recognition of educational activities only; accredited status does not imply endorsement by NCCN or ANCC of any commercial products discussed/displayed in conjunction with the educational activity. Kristina M. Gregory, RN, MSN, OCN, is our nurse planner for this educational activity.

All clinicians completing this activity will be issued a certificate of participation. To participate in this journal CE activity: 1) review the learning objectives and author disclosures; 2 ) study the education content; 3 ) take the posttest with a $66 \%$ minimum passing score and complete the evaluation at http://education.nccn.org/ node/35439; and 4) view/print certificate.

Release date: December 16, 2013; Expiration date: December 16, 2014

\section{Learning Objectives}

Upon completion of this activity, participants will be able to:

- Explain factors to consider when deciding whether to perform APC testing for possible FAP

- Summarize appropriate criteria for tumor testing for possible Lynch syndrome in patients with colorectal cancer

- Discuss the potential role of a universal testing strategy (UTS) for Lynch syndrome

EDITOR

Kerrin M. Green, MA, Assistant Managing Editor, JNCCN_Journal of the National Comprehensive Cancer Network

Ms. Green has disclosed that she has no relevant financial relationships.

\section{CE AUTHORS}

Deborah J. Moonan, RN, BSN, Manager, CE Supporter Outreach

Ms. Moonan has disclosed the following relationship with commercial interests: AstraZeneca: Stockholder/Former Employee.

Ann Gianola, MA, Manager, Medical Education Accreditation and Grant Development

Ms. Gianola has disclosed the following relationship with commercial interests: Actelion: Grant/Research Support.

Kristina M. Gregory, RN, MSN, OCN, Vice President, Clinical Information Operations

Ms. Gregory has disclosed that she has no relevant financial relationships. 
The 2013 NCCN Clinical Practice Guidelines in Oncology (NCCN Guidelines) for Colorectal Cancer (CRC) Screening address the evaluation and management of patients at risk of inherited susceptibility to colorectal cancer (available in this issue; to view the most recent version of these guidelines, visit NCCN.org). ${ }^{1}$ These patients include the 2 broad categories of patients with familial adenomatous polyposis (FAP) and its variants, and those with hereditary nonpolyposis CRC (HNPCC), otherwise known as Lynch syndrome (LS). This commentary provides a somewhat personal, yet hopefully evidence-based, approach to the questions of when to test individuals for inherited susceptibility, and how to do so. "When" can be taken to mean "under what circumstance" and "at what age"; this article attempts to speak to each sense of the term.

The questions a clinician must consider initially are: 1) what disease process am I entertaining?, 2) is there a potentially informative genetic test?, and 3) has that test already been performed on this patient or a family member and, if so, was it informative?

\section{What Is the Disease Process in Question?}

This may be very straightforward, as in most cases of FAP. The patient may have a clinical diagnosis of hundreds of adenomas, which is diagnostic in and of itself. In this case, genetic testing will not really alter the patient's management, but will serve as a basis for predictive testing in at-risk relatives. ${ }^{2-4}$ More recently, patients have been noted to have oligopolyposis $(<100$ polyps, often $<10$ or 20$) ..^{5}$ This patient may have attenuated FAP with a mutation in the APC gene. ${ }^{6}$ Biallelic mutations in the (recessive) MUTYH gene may be found instead, and the counseling clinician must be prepared to offer both APC and MUTYH testing. ${ }^{7}$ In these cases, detecting a mutation will both confirm the clinical diagnosis and, as in the case of more classic FAP, serve as a foundation for predictive counseling and testing in other family members.

As the NCCN Guidelines for CRC Screening ${ }^{1}$ continue to emphasize, a patient with FAP or a variant is asked at the initial assessment whether mutational testing has been performed. If the nature of the polyposis is straightforward, with the patient clinically affected, and no one else was already tested, then testing is offered. This involves full-length sequencing of the APC gene, rearrangement studies in the event of a large deletion not detectable on sequencing, ${ }^{8}$ and possibly MUTYH gene testing. This is an expensive undertaking. Ideally, when considering testing of an unaffected at-risk patient, it should involve an effort to detect the presence or absence of a mutation already identified in another family member. This is especially important when the patient in question is young and not known to be affected with adenomas, and being evaluated for the presence of mutation before initiation of clinical screening. In these cases, the counselor attempts to find an affected parent or other relative for initial testing to confirm the presence of a pathologic family mutation, which should be present in up to $90 \%$ of families with classic FAP.

Generally, APC testing is not offered before the age of approximately 10 to 12 years, which is the age at which clinical surveillance for polyps would otherwise be appropriate. An argument can be made for testing in infancy when risk of hepatoblastoma is a concern. ${ }^{9}$ When no mutation is found in an affected relative, those at risk must undergo clinical surveillance "as if" they were mutation carriers, because in this circumstwance they cannot be proven not to be carriers. If the affected parent is deceased and no mutation information is present, several options may be considered. Ideally, some other affected relative, perhaps a sibling of the deceased parent, has been tested or is available for testing. If no known affected individual is available, the young, at-risk patient can be tested. However, in this setting, a negative test remains nondiagnostic (indeterminate or uninformative), because approximately $10 \%$ or more of cases of even classic FAP will have uninformative testing. If additional at-risk siblings are tested, and any are found to carry an APC mutation, then the previous nondiagnostic test can be reclassified as a true-negative. Although the Bayesian principles of Mendelian genetics involved in this process are straightforward, providing formal genetic counseling is nevertheless advisable in most instances, lest missteps in the testing process occur. ${ }^{10,11}$

As suggested earlier, the clinical diagnosis of FAP may itself be in serious question. The adenoma burden may be relatively light: fewer than 50 polyps, maybe fewer than 10 or 20 . The patient may be older at diagnosis ( $\geq 40$ years of age) and/or have no known family history of similar polyp burden. When 
the polyps are few or occur late, APC testing may confirm the diagnosis, although polyposis in additional affected relatives may confirm the diagnosis at a clinical level. In the case of this more attenuated FAP, mutational testing is less likely to be diagnostic than in classic FAP, with APC mutation detection ranging from $56 \%$ in those with 100 to 999 adenomas (a likely mix of classic and attenuated FAP) to as low as $10 \%$ when only 10 to 99 adenomas are present. ${ }^{12}$ When the polyp burden is low and the family history is negative, there may be an alternate genetic diagnosis, namely biallelic mutations in the MUTYH gene. ${ }^{13}$ Biallelic mutations in this recessively acting gene account for approximately $10 \%$ to $20 \%$ of cases of polyposis occurring in the absence of parental involvement. ${ }^{14}$ As expected, one-fourth of siblings can be expected to be clinically affected. A modest increase in adenoma and cancer risk seems to be present in patients with only one mutated MU TYH allele, but the magnitude of this risk appears to be low. ${ }^{15}$

\section{When to Test for Suspected LS?}

Often the most challenging case will be the patient with CRC but few or no adenomas. Typically, a patient such as this will have a CRC detected based on symptoms or screening colonoscopy, possibly prompted by a reported family history of CRC. If the patient is young and/or the tumor is right-sided, and especially in the presence of a positive family history, the presence of LS should be suspected. ${ }^{16-19}$

As in the case of FAP, the approach to evaluating for LS depends on whether genetic testing has already been performed on someone else in the family, and what that testing yielded. As time passes, increasingly more patients with CRC will have undergone some form of testing, and so the chance that some other relative has already been evaluated can only increase in the future. Therefore, it is always helpful, as part of the general medical history, to not only ask about the family history of cancer but also ask whether any relatives with colorectal, endometrial, or other LS spectrum tumors have already been tested. Unfortunately, it is still the exceptional case in which this evaluation has already been performed. Furthermore, if it has been performed, a concerned, at-risk relative will not always arrive in the clinic armed with the pertinent information. Retrieving the results of the previously conducted genetic test from the laboratory report is helpful to determine whether the result was informative.

When no antecedent testing in the family is available to rely on, the questions may include the following:

- Has the patient been diagnosed with CRC?

- When malignant tissue is not available, can a benign adenoma be evaluated for microsatellite instability (MSI) or loss of mismatch repair (MMR) protein expression through immunohistochemistry (IHC) $?^{20} \mathrm{IHC}$ and MSI testing have not been validated in colorectal adenomas, even though they are performed by some clinicians.

- Should practitioners always follow clinical guidelines for tumor testing, such as Bethesda Guidelines, or is the institution one of the growing number in which such MSI and/or IHC testing is performed "universally"? ${ }^{21,22}$ A universal testing strategy (UTS) is described later.

- If the patient has no cancer diagnosis and the consultation is undertaken because of concerning family history only, is the a priori risk high enough to warrant germline mutation testing anyway ${ }^{16}$

\section{Has the Patient Been Diagnosed With CRC, and if So, What Is the Appropriate Threshold for Tumor Testing?}

MSI, when present and not caused by methylation of the MLH1 promoter (see later discussion), is a strong predictor of LS, and generally warrants germline mutation testing. ${ }^{23-30}$ Conversely, a normal MSI/IHC constitutes a very strong negative predictor, such that mutation testing in the face of normal MSI/IHC should only be considered when the clinical picture is otherwise compelling. Current NCCN Guidelines for CRC Screening ${ }^{1}$ (in this issue) recommend tumor testing when any of the Bethesda Guidelines ${ }^{29,30}$ criteria are met (to view subsequent updates to these guidelines, visit NCCN.org). In light of increasing adoption of UTS, the new NCCN Guidelines include the option of UTS as an alternative to use of Bethesda Guidelines. ${ }^{1,2,31}$ These recommendations were proposed in order to have a threshold for MSI tumor testing that was reasonably sensitive and specific for detecting likely candidates for the subsequent step of mutational testing. The guidelines essentially 
propose tumor testing in any patients diagnosed with CRC before 70 years of age, and for testing after that age when varying combinations of other clinical features are present, such as family history or multiple primary cancers. Before 50 years of age, a tumor with MSI-whether detected through PCR-based methodology or the common surrogate, IHC involving antibodies against the proteins corresponding to the several MMR genes ${ }^{31-34}$ — will indicate a high likelihood of HNPCC/LS. In older patients with a modest or absent family history, an abnormal MSI or IHC result may indicate a sporadic, epigenetically, or somatically acquired defect. ${ }^{23-25}$ The finding may still be important. In these cases, an assay directed at detecting MLH-1 hypermethylation or its surrogate, BRAF mutation, may suggest the non-HNPCC/LS basis and a recommendation for no further HNPCC/ LS-related testing. MSI/IHC testing is now often routine and does not require either a written informed consent or any formal genetic counseling. However, when testing follows such a routine, it is nevertheless essential that the institution have oversight of the process. This includes proficiency testing that assesses the correlation between MSI and IHC findings, which should be at least $95 \%$, because errors in interpretation could occur, especially involving weak or patchy IHC staining. ${ }^{35,36}$ Furthermore, an experienced genetic counselor and clinician should review the findings of both informative and uninformative cases to ensure that results of individual cases and the larger practice are consistent with expected values. Periodic updates of institutional performance are warranted for all involved clinicians. ${ }^{21}$

Tumor testing need not be limited to CRC. Routine evaluation of endometrial cancers for MSI is emerging, and MSI testing does seem to be potentially informative for other extracolonic cancers within the HNPCC/LS spectrum. ${ }^{37-39}$

\section{Can Adenomas Be Evaluated for MSI/Abnormal IHC?}

In some patients, particularly those undergoing colonic surveillance based on a positive family history, the only pertinent finding may be a premalignant adenoma. If no malignancy is available for testing, and an a priori risk model does not predict a high likelihood of mutation, an adenoma can be tested for MSI/IHC. ${ }^{20,40}$ It has been shown that even in pa- tients with proven MMR mutations, the likelihood of MSI being informative in adenomas is considerably lower than in malignancy. Not surprisingly, the yield increases with adenoma size and severity of dysplasia. ${ }^{20,40}$ Therefore, it may be worth testing an adenoma, but normal findings do not carry the negative predictive power that normal findings in a cancer would. Finding an adenoma in a young patient with a strong family history may support a case for germline mutation testing. However, existing data and the corresponding risk-assessment models have not really been able to provide any appropriate support for mutation testing in the presence of an adenoma only, at least not in the absence of MSI.

Rectal cancers require some comment. Rectal cancer is relatively more common in patients with MSH6 mutations, ${ }^{40,41}$ but can be seen in patients with other MMR mutations. Most patients with rectal cancer present with locally advanced disease, for which preoperative chemoradiation is typically given. Commonly, little or no residual tumor remains after this treatment, and therefore the informativeness of MSI testing will be reduced. ${ }^{42,43}$ For this reason, obtaining adequate tumor tissue before chemoradiation is ideal. In fact, performance of MSI/IHC at the initial CRC diagnosis is helpful even in patients for whom preoperative chemoradiation is not planned, because an informative MSI/IHC before surgery may affect the approach (extent) of surgery, even if germline testing has not yet been performed or is otherwise nondiagnostic.

\section{Role for a UTS}

Many institutions are now adopting a UTS for all new CRCs to determine evidence of MSI, rather than basing the decision to evaluate on some clinical subsets, such as the Bethesda Guidelines. ${ }^{21,22}$ The rationale is 2-fold: 1) even liberal application of the Bethesda Guidelines fails to detect some cases of HNPCC/LS, and 2) recognizing that no more than approximately $20 \%$ of MSI CRCs in fact have HNPCC/LS, and the rest have acquired MLH1 promoter methylation-mediated MSI, then any case of MSI may be worth detecting because of differences in prognosis and potential response to chemotherapeutic agents. ${ }^{43}$ Current NCCN Guidelines for CRC Screening $^{1}$ (in the issue) do recommend this as one option, with the alternative being testing the tumors 
Genetic Testing for Inherited CRC Syndromes

from patients younger than 70 years and older patients when additional Bethesda features are present (to view subsequent updates to these guidelines, visit NCCN.org). A truly universal approach has been more positively endorsed by the Evaluation of Genomic Applications in Practice and Prevention (EGAPP) working group. It is too early to tell whether this will become a more standard practice, but the trend seems to moving in this direction. ${ }^{31}$

Another even more ambitious approach involves the use of very large panels of genes that have had exomic sequencing performed via next-generation sequencing technology. ${ }^{44,45}$ These high-throughput approaches can be used to identify mutations in tumors that may suggest differences in prognosis and treatment responsiveness, but on a much larger (and unfocused) scale than the adoption of UTS. These multigene panels commonly include MLH1 and one or more of the other MMR genes, so at least some potential HNPCC/LS cases would be routinely identified.

\section{Germline Mutation Testing}

However targeted, patients undergoing germline mutation testing for HNPCC/LS, and their providers, must accept the fact that current clinical testing identifies pathogenic mutations in only approximately $50 \%$ to $70 \%$ of patients. ${ }^{28,46}$

This testing generally includes both sequencing of exons in exon-intron boundaries, along with an assay for the comprehensive detection of large rearrangements.

Current NCCN Guidelines for CRC Screening ${ }^{1}$ (in this issue) generally assume that an index patient will be one with cancer who has a tumor available for MSI testing, whether this has followed a clinical selection strategy or the newer UTS (to view subsequent updates to these guidelines, visit NCCN. org). However, the guidelines do provide for the situation in which no tumor is present. The typical situation is the healthy adult child of a parent who is deceased and whose tumor diagnosis was more than 10 years ago, such that tumor blocks are no longer available for MSI/IHC. Dinh et $\mathrm{al}^{47}$ performed analyses that used risk-assessment models (in this case the PREMM ${ }_{1,2,6}$ model developed at the DanaFarber Cancer Institute ${ }^{48}$ ) to estimate thresholds for cost-effectiveness in testing. Using the very simple- to-administer PREMM model, prior probabilities of $5 \%$ or greater in young adults were considered an appropriate threshold for considering mutational testing. This practice has not gained widespread acceptance in decision-making about testing, given the more powerful positive and negative predictive power of MSI-based testing. However, the use of riskassessment models has, in the author's experience, proven very helpful in framing the probabilities of mutation detection for individual patients.

\section{Conclusions}

Current NCCN Guidelines for CRC Screening ${ }^{1}$ (in this issue) provide a detailed approach to decisionmaking with respect to patients suspected of inherited susceptibility to multiple polyp disorders and also to HNPCC/LS (to view subsequent updates to these guidelines, visit NCCN.org). Care is taken to properly characterize the clinical presentation of a given subject, including details regarding family history. This enables testing for the most appropriate genes. At the same time, current algorithmic approaches recognize the limitations of genetic testing, mainly the possibility that this testing may fail to identify a mutation. This in turn requires that clinically oriented decision-making include strategies for cancer surveillance in the event of nondiagnostic testing.

In the polyposis-related disorders, whether adenomatous or hamartomatous, it is generally easy to determine that a patient is affected, and the yield in mutational testing of these individuals is high. In the uncommon case of uninformative genetic testing, endoscopic or other screening of at-risk relatives remains fairly straightforward, as described in the NCCN algorithms. ${ }^{1}$

HNPCC/LS poses somewhat greater complexity and challenge. It may be difficult to be sure that a given patient with cancer is syndromically "affected" at all, and tumor MSI and/or family history may or may not be compelling. Even in a "best case" scenario of MSI-high status (or protein loss when IHC used) and a compelling clinical picture, the mutation detection rate rarely exceeds $70 \%$. When a mutation is found, testing of patients who are at-risk is straightforward, but the clinical screening of carriers can be daunting, expensive, and potentially risky. Despite these various pitfalls, the current NCCN Guidelines for CRC Screening ${ }^{1}$ (in this issue) pro- 
vide a helpful guide for clinicians willing to walk patients through the process (to view subsequent updates to these guidelines, visit NCCN.org). As in all such endeavors, reliance on properly trained genetic counselors $^{49}$ can help assure that the patient is wellinformed of testing-related issues. This includes the need to alert at-risk relatives of the potential benefits of a mutation testing-driven approach to their own management, rather than have them undergo purely empiric screening that is based only on the "positive family history."

\section{References}

1. Burt RW, Cannon JA, David DS, et al. NCCN Clinical Practice Guidelines in Oncology: Colorectal Cancer Screening, Version 2, 2013. Available at: NCCN.org. Accessed October 30, 2013.

2. Petersen GM, Brensinger JD, Johnson KA, Giardiello FM. Genetic testing and counseling for hereditary forms of colorectal cancer. Cancer 1999;86(11 Suppl):2540-2550.

3. Giardiello FM, Brensinger JD, Petersen GM, et al. The use and interpretation of commercial APC gene testing for familial adenomatous polyposis. N Engl J Med 1997;336:823-827.

4. Giardiello FM, Brensinger JD, Petersen GM. AGA technical review on hereditary colorectal cancer and genetic testing. Gastroenterology 2001;121:198-213.

5. Leppert M, Burt R, Hughes JP, et al. Genetic analysis of an inherited predisposition to colon cancer in a family with a variable number of adenomatous polyps. N Engl J Med 1990;322:904-908.

6. Spirio L, Otterud B, Stauffer D, et al. Linkage of a variant or attenuated form of adenomatous polyposis coli to the adenomatous polyposis coli (APC) locus. Am J Hum Genet 1992;51:92-100.

7. Church J, Heald B, Burke C, Kalady M. Understanding MYHassociated neoplasia. Dis Colon Rectum 2012;55:359-362.

8. Meuller J, Kanter-Smoler G, Nygren AO, et al. Identification of genomic deletions of the APC gene in familial adenomatous polyposis by two independent quantitative techniques. Genet Test 2004;8:248-256.

9. Aretz S, Koch A, Uhlhaas S, et al. Should children at risk for familial adenomatous polyposis be screened for hepatoblastoma and children with apparently sporadic hepatoblastoma be screened for APC germline mutations? Pediatr Blood Cancer 2006;47:811-818.

10. Trimbath JD, Giardiello FM. Review article: genetic testing and counselling for hereditary colorectal cancer. Aliment Pharmacol Ther 2002;16:1843-1857.

11. Markey K, Axel L, Ahnen D. Basic concepts for genetic testing in common hereditary colorectal cancer syndromes. Curr Gastroenterol Rep 2002;4:404-413.

12. Grover S, Kastrinos F, Steyerberg EW, et al. Prevalence and phenotypes of APC and MUTYH mutations in patients with multiple colorectal adenomas. JAMA 2012;308:485-492.

13. Al-Tassan $\mathrm{N}$, Chmiel NH, Maynard J, et al. Inherited variants of MYH associated with somatic G:C-->T:A mutations in colorectal tumors. Nat Genet 2002;30:227-232.

14. Sampson JR, Dolwani S, Jones S, et al. Autosomal recessive colorectal adenomatous polyposis due to inherited mutations of MYH. Lancet 2003;362:39-41.

15. Croitoru ME, Cleary SP, Di Nicola N, et al. Association between biallelic and monoallelic germline MYH gene mutations and colorectal cancer risk. J Natl Cancer Inst 2004;96:1631-1634.
16. Kastrinos F, Steyerberg EW, Balmana J, et al. Comparison of the clinical prediction model PREMM $(1,2,6)$ and molecular testing for the systematic identification of Lynch syndrome in colorectal cancer. Gut 2013;62:272-279.

17. Ladabaum U, Ford JM. Lynch syndrome in patients with colorectal cancer: finding the needle in the haystack. JAMA 2012;308:1581-1583.

18. Moreira L, Balaguer F, Lindor N, et al. Identification of Lynch syndrome among patients with colorectal cancer. JAMA 2012;308:1555-1565.

19. Lynch PM. Current approaches in hereditary nonpolyposis colorectal cancer. J Natl Compr Canc Netw 2012;10:961-967.

20. Yurgelun MB, Goel A, Hornick JL, et al. Microsatellite instability and DNA mismatch repair protein deficiency in Lynch syndrome colorectal polyps. Cancer Prev Res (Phila) 2012;5:574-582.

21. Heald $B$, Plesec $T$, Liu $X$, et al. Implementation of universal microsatellite instability and immunohistochemistry screening for diagnosing lynch syndrome in a large academic medical center. J Clin Oncol 2013;31:1336-1340.

22. Byfield SA, Syngal S. Clinical guidelines versus universal molecular testing: are we ready to choose an optimal strategy for Lynch syndrome identification? Am J Gastroenterol 2008;103:2837-2840.

23. Parsons MT, Buchanan DD, Thompson B, et al. Correlation of tumour BRAF mutations and MLH1 methylation with germline mismatch repair (MMR) gene mutation status: a literature review assessing utility of tumour features for MMR variant classification. J Med Genet 2012;49:151-157.

24. Gausachs M, Mur P, Corral J, et al. MLH1 promoter hypermethylation in the analytical algorithm of Lynch syndrome: a cost-effectiveness study. Eur J Hum Genet 2012;20:762-768.

25. Perez-Carbonell L, Alenda C, Paya A, et al. Methylation analysis of MLH1 improves the selection of patients for genetic testing in Lynch syndrome. J Mol Diagn 2010;12:498-504.

26. Evaluation of Genomic Applications in Practice and Prevention (EGAPP) Working Group. Recommendations from the EGAPP Working Group: genetic testing strategies in newly diagnosed individuals with colorectal cancer aimed at reducing morbidity and mortality from Lynch syndrome in relatives. Genet Med 2009;11:35-41.

27. Hampel H, Frankel WL, Martin E, et al. Screening for the Lynch syndrome (hereditary nonpolyposis colorectal cancer). N Engl J Med 2005;352:1851-1860.

28. Pinol V, Castells A, Andreu M, et al. Accuracy of revised Bethesda guidelines, microsatellite instability, and immunohistochemistry for the identification of patients with hereditary nonpolyposis colorectal cancer. JAMA 2005;293:1986-1994.

29. Rodriguez-Bigas MA, Boland CR, Hamilton SR, et al. A National Cancer Institute Workshop on Hereditary Nonpolyposis Colorectal Cancer Syndrome: meeting highlights and Bethesda guidelines. J Natl Cancer Inst 1997;89:1758-1762.

30. Umar A, Boland CR, Terdiman JP, et al. Revised Bethesda Guidelines for hereditary nonpolyposis colorectal cancer (Lynch syndrome) and microsatellite instability. J Natl Cancer Inst 2004;96:261-268.

31. Beamer LC, Grant ML, Espenschied CR, et al. Reflex immunohistochemistry and microsatellite instability testing of colorectal tumors for Lynch syndrome among US cancer programs and follow-up of abnormal results. J Clin Oncol 2012;30:10581063.

32. Mojtahed A, Schrijver I, Ford JM, et al. A two-antibody mismatch repair protein immunohistochemistry screening approach for colorectal carcinomas, skin sebaceous tumors, and gynecologic tract carcinomas. Mod Pathol 2011;24:1004-1014.

33. Zhang L. Immunohistochemistry versus microsatellite instability testing for screening colorectal cancer patients at risk for hereditary nonpolyposis colorectal cancer syndrome. Part II. 
Genetic Testing for Inherited CRC Syndromes

The utility of microsatellite instability testing. J Mol Diagn 2008; 10:301-307.

34. Shia J. Immunohistochemistry versus microsatellite instability testing for screening colorectal cancer patients at risk for hereditary nonpolyposis colorectal cancer syndrome. Part I. The utility of immunohistochemistry. J Mol Diagn 2008;10:293-300.

35. Samowitz WS, Broaddus R, Iacopetta B, et al. PCR versus immunohistochemistry for microsatellite instability. J Mol Diagn 2008;10:181-182; author reply 181.

36. Watson N, Grieu F, Morris M, et al. Heterogeneous staining for mismatch repair proteins during population-based prescreening for hereditary nonpolyposis colorectal cancer. J Mol Diagn 2007;9:472-478.

37. Lu KH, Schorge JO, Rodabaugh KJ, et al. Prospective determination of prevalence of lynch syndrome in young women with endometrial cancer. J Clin Oncol 2007;25:5158-5164.

38. Broaddus RR, Lynch PM, Lu KH, et al. Unusual tumors associated with the hereditary nonpolyposis colorectal cancer syndrome. Mod Pathol 2004;17:981-989.

39. Abbas $\mathrm{O}$, Mahalingam M. Cutaneous sebaceous neoplasms as markers of Muir-Torre syndrome: a diagnostic algorithm. J Cutan Pathol 2009;36:613-619.

40. Klarskov L, Holck S, Bernstein I, et al. Challenges in the identification of MSH6-associated colorectal cancer: rectal location, less typical histology, and a subset with retained mismatch repair function. Am J Surg Pathol 2011;35:1391-1399.

41. Plaschke J, Engel C, Kruger S, et al. Lower incidence of colorectal cancer and later age of disease onset in 27 families with pathogenic MSH6 germline mutations compared with families with MLH1 or MSH2 mutations: the German Hereditary Nonpolyposis
Colorectal Cancer Consortium. J Clin Oncol 2004;22:44864494.

42. Bao F, Panarelli NC, Rennert H, et al. Neoadjuvant therapy induces loss of MSH6 expression in colorectal carcinoma. Am J Surg Pathol 2010;34:1798-1804.

43. Sinicrope FA, Foster NR, Thibodeau SN, et al. DNA mismatch repair status and colon cancer recurrence and survival in clinical trials of 5-fluorouracil-based adjuvant therapy. J Natl Cancer Inst 2011;103:863-875.

44. Han SW, Kim HP, Shin JY, et al. Targeted sequencing of cancer-related genes in colorectal cancer using next-generation sequencing. PLoS One 2013;8:e64271.

45. Garraway LA, Verweij J, Ballman KV. Precision oncology: an overview. J Clin Oncol 2013;31:1803-1805.

46. Mangold E, Pagenstecher C, Friedl W, et al. Spectrum and frequencies of mutations in MSH2 and MLH1 identified in 1,721 German families suspected of hereditary nonpolyposis colorectal cancer. Int J Cancer 2005;116:692-702.

47. Dinh TA, Rosner BI, Atwood JC, et al. Health benefits and costeffectiveness of primary genetic screening for Lynch syndrome in the general population. Cancer Prev Res (Phila) 2011;4:9-22.

48. Kastrinos F, Steyerberg EW, Balmana J, et al. Comparison of the clinical prediction model PREMM ${ }_{1,2,6}$ and molecular testing for the systematic identification of Lynch syndrome in colorectal cancer. Gut 2013;62:272-279.

49. Weissman SM, Burt R, Church J, et al. Identification of individuals at risk for Lynch syndrome using targeted evaluations and genetic testing: National Society of Genetic Counselors and the Collaborative Group of the Americas on Inherited Colorectal Cancer Joint Practice Guideline. J Genet Couns 2012;21:484493.

\section{Instructions for Completion}

To participate in this journal CE activity: 1) review the learning objectives and author disclosures; 2 ) study the education content; 3 ) take the posttest with a $66 \%$ minimum passing score and complete the evaluation at http://education.nccn.org/ node/35439; and 4) view/print certificate. After reading the article, you should be able to answer the following multiple- choice questions. Credit cannot be obtained for tests completed on paper. You must be a registered user on NCCN.org. If you are not registered on NCCN.org, click on "New Member? Sign up here" link on the left hand side of the Web site to register. Only one answer is correct for each question. Once you successfully answer all posttest questions you will be able to view and/or print your certificate. Software requirements: Internet

\section{PostTest Questions}

1. A 12-year-old patient with a family history of FAP presents at your clinic. Which of the following must be present before using APC testing?

a. The presence of a known pathologic family mutation

b. The known presence of adenomas in the patient

c. Both $a$ and $b$.

d. None of the above.

2. True or False: Germline mutation testing for HNPCC/LS identifies pathogenic mutations in only approximately $50 \%$ to
$70 \%$ of patients.

a. True

b. False

3. Genetic testing in a patient with a clinical diagnosis of FAP accomplishes which of the following?

a. Determines the patient's management

b. Serves as a basis for predictive testing in at-risk relatives

c. All of the above

d. None of the above 